\title{
Theoretical analysis of the liquid thermal structure in a pool fire
}

\section{Tarek Beji}

\begin{abstract}
The paper presents a theoretical work on liquid heat-up in the case of a pool fire. It is assumed that the convective currents occurring within the upper layer of the liquid are induced by Rayleigh-Bénard instabilities that are caused by in-depth radiation. The upper layer depth has been estimated based on the analytical solution of a 1-D Fourier's equation for the temperature with a source term for in-depth radiation. The model has been assessed against experimental data for a $9 \mathrm{~cm}$ - diameter methanol steady-state pool fire and three different liquid depths $(18,12$ and $6 \mathrm{~mm}$ ). The general trend, i.e., increase in the upper layer depth as the bottom boundary temperature increases, is well captured. In order to ensure that the well-mixed upper layer is at a temperature near the boiling point (as suggested by the experimental data), an improvement is proposed based on a radiative heat balance integral method. In addition to the above, a novel methodology is developed for the calculation of the 'effective' thermal conductivity as a means to circumvent detailed calculations of heat transfer within the liquid.
\end{abstract}

Keywords: Liquid pool fire; heat transfer; in-depth radiation; analytical modelling. 


\section{Introduction}

Quite extensive research is carried out on the numerical modelling of liquid pool fires, given the potential hazard induced by these types of fires. More specifically, Computational Fluid Dynamics (CFD) is an advanced technique that is extensively being used for the simulation of pool fires. However, the current developments and applications are mostly devoted to gas phase aspects, e.g., combustion and thermal radiation modelling [1]. In this case, the burning (evaporation) rate is prescribed according to an experimentally measured value or an estimate based on a semi-empirical correlation. In reality, the burning rate is strongly coupled to the heat feedback from the flame (and, eventually, other 'external sources' such as the smoke layer, ceiling and walls in an enclosure fire). The higher the heat feedback, the higher the burning rate (because of a more intense liquid heat-up and evaporation) and vice versa. Therefore, in order to perform fully predictive simulations of liquid pool fires, the coupling liquid phase-gas phase must be considered. The most advanced approach would be to solve the full set of Navier-Stokes equations for the liquid phase (as it is the case for the gas phase), as recently attempted in [2]. However, a more thorough and extensive assessment of this approach is still required. Furthermore, such approach is believed to require prohibitive amounts of computational resources for 'practical' fire dynamics simulations, considering the resources that would have been already allocated for the gas phase. A significantly simpler approach consists of solely solving the heat-up of the liquid layer, and not the momentum equation therein, using a 1-D Fourier's equation, as if it were a thermally-thick solid. By doing so, the convective currents in liquid fuels during pool burning, as pointed out in [2-3], are neglected. The idea is then to model 'indirectly' the convective currents, keeping the 1-D Fourier's equation as a general framework. This has been mainly developed in [3] where the main assumption is that 'in large pool fires the main source of convective motions is buoyancy-generated by in-depth radiation absorption'. Therefore, the work presented in [3] is mainly centered around two aspects: (1) the modelling of a radiative source term in the heat conduction equation, including the estimation of the absorption coefficients of liquids, and (2) the modelling of an 'effective thermal conductivity', which has been referred to above as an 'indirect' modelling of convective currents that enhance heat transfer within the liquid. As opposed to [3], the purpose of the present work is not to implement the proposed sub-models in CFD simulations and assess the latter against transient profiles and steady-state values of the burning rate for a wide range of liquids and pan 
diameters. The intent is rather to take a step back and focus specifically on the thermal structure of the liquid and the modelling of an 'effective' thermal conductivity coefficient. The assessment of the proposed work is based on the experimental profiles of liquid temperature provided in [4], bearing in mind that some of the profiles discussed in [3] were 'clearly unphysical'.

\section{Experimental data}

To the best of the author's knowledge, the most comprehensive experimental work on heat transfer within the liquid in a pool fire is described in [4] for a $9 \mathrm{~cm}$ - diameter methanol steadystate pool fire and three different liquid depths (18, 12 and $6 \mathrm{~mm})$, maintaining the bottom boundary temperature at several values between -10 and $50^{\circ} \mathrm{C}$. More specifically, the velocity field within the liquid fuel was determined by Particle Image Velocimetry (PIV) and the temperature was measured by type $\mathrm{K}$ thermocouple probes. A distinct two-layer thermal structure was depicted and estimates of the thickness of the lower layer are provided in [4]. Given the fact that in [4] a steady fuel level was maintained in the pool, the thickness of the top layer can be easily retrieved from the data displayed therein. The uniform temperature within the vaporizing layer was attributed to two-counter rotating vortices. The first vortex, close to the pool wall, is explained by the 'buoyancy force near the pool wall and shear stress forces at the liquid-gas interface'. Several potential reasons are provided for the development of the second vortex, e.g., 'the liquid-gas interface shear force' or the 'non-uniformity of local evaporation rate at the pool surface'. In [5], it is stated that in-depth (radiation) absorption leads to 'in-depth temperatures' higher than the surface temperature, generating thus a convective current (due to Rayleigh convection) that drives the mixing within the boiling layer. This phenomenon causes the temperature profile across the vaporizing fuel layer to be uniform. Rayleigh convection has been confirmed experimentally in [5] using holographic interferometry for a toluene pool fire. Generally speaking, the two-layer thermal structure described in [4] is suitable for 'thin' pools. A more 'complete' description of the liquid thermal structure is provided in [6] where, for sufficiently 'deep pools', beneath the 'preheating layer', there is 'bottom fuel layer' where the fuel temperature does not vary substantially, see Fig. 1. 


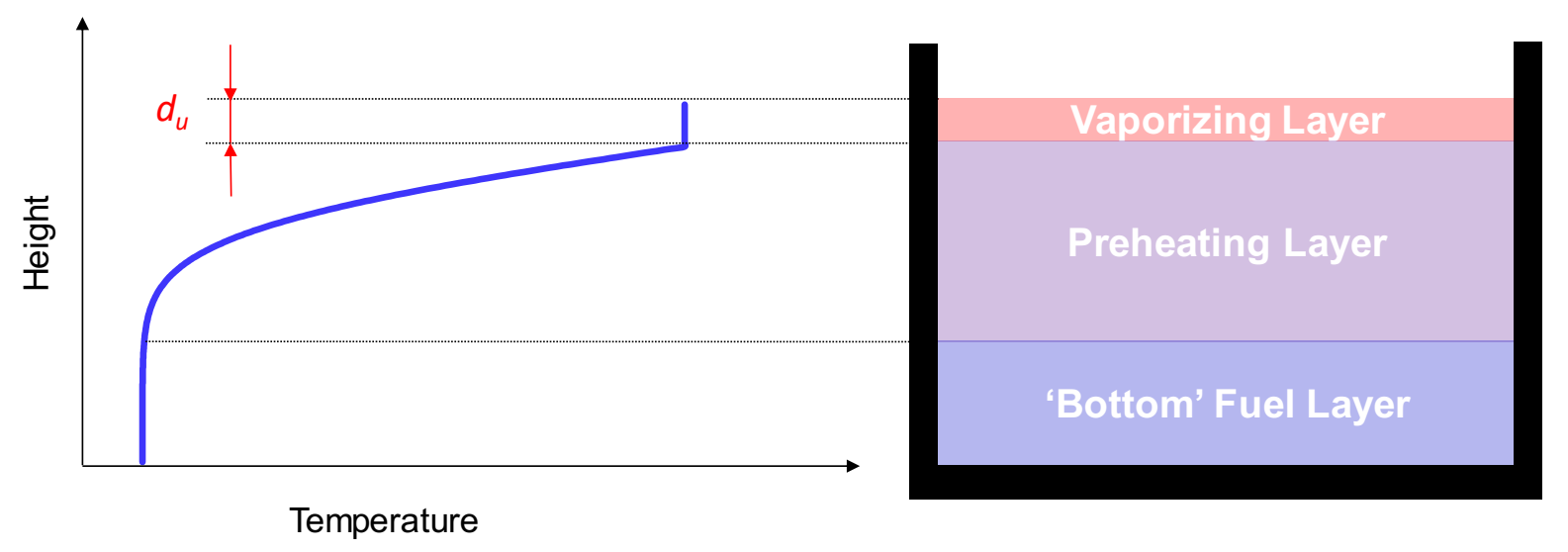

Figure 1 - Thermal structure within the liquid in the case of a pool fire.

Based on the thermal structure displayed in Fig. 1, for 'thin' pools (as in [4]) the upper layer is the vaporizing layer and the lower layer is the preheating layer.

\section{Numerical modelling}

The numerical simulation of in-depth radiation has been undertaken in $[5,7]$ by considering a source term in the governing energy equation (for the liquid) where the radiative flux at a given depth within the liquid is derived by applying the 'classical attenuation law', i.e., Beer - Lambert law, to the radiative heat flux at the fuel surface using a mean (spectrally-averaged) absorption coefficient. As explained in [7], this approach generally leads to a 'temperature inversion layer' that is not observed experimentally. This is explained in [7] by the fact that 'the onset of convective currents (Rayleigh effect) generated by the radiation absorption near the surface [is] not considered in the theoretical model'. This has been one of the main modelling aspects addressed in [3] and which will be recalled in the following sub-section in order to put the present work into context and better highlight the novelties with respect to the developments proposed in [3].

\subsection{Approach of Sikanen and Hostikka [3]}

In [3], 'the one-dimensional heat conduction equation for liquid temperature is applied in the $x$ direction pointing into the liquid (the point $x=0$ represents the surface)' and it reads: 
$\rho c \frac{\partial T(x, t)}{\partial t}=\frac{\partial}{\partial x}\left(k \frac{\partial T(x, t)}{\partial x}\right)+\dot{q}_{r}^{\prime \prime}$

where $\rho, k, c$ and $T$ are respectively the density, the thermal conductivity, the specific heat and the temperature of the liquid. The variables $t$ and $\dot{q}_{r}^{\prime \prime \prime}$ denote the time and the volumetric radiative heat source term within the liquid, respectively. The thermal boundary condition on the top surface of the liquid reads:

$-k \frac{\partial T}{\partial x}(0, t)=\dot{q}_{c, s}^{\prime \prime}+\dot{q}_{r, s}^{\prime \prime}-L_{v} \dot{m}^{\prime \prime}$

where $\dot{q}_{c, s}^{\prime \prime}$ and $\dot{q}_{r, s}^{\prime \prime}$ are respectively the convective and radiative heat fluxes at the surface, $L_{v}$ is the latent heat of vaporization and $\dot{m}$ "is the evaporation rate. In case of internal radiation, the net radiative heat flux at the surface should be set to zero, as pointed out in [3].

The key parameters of interest here are $k, \dot{q}_{r}^{\prime \prime \prime}$ and $\dot{q}_{r, s}^{\prime \prime}$.

The volumetric radiative heat source term in the heat conduction equation is calculated in [3] by solving the transport of heat radiation inside the liquid layer using a 'two-flux' model based on the Schuster-Schwarzschild approximation.

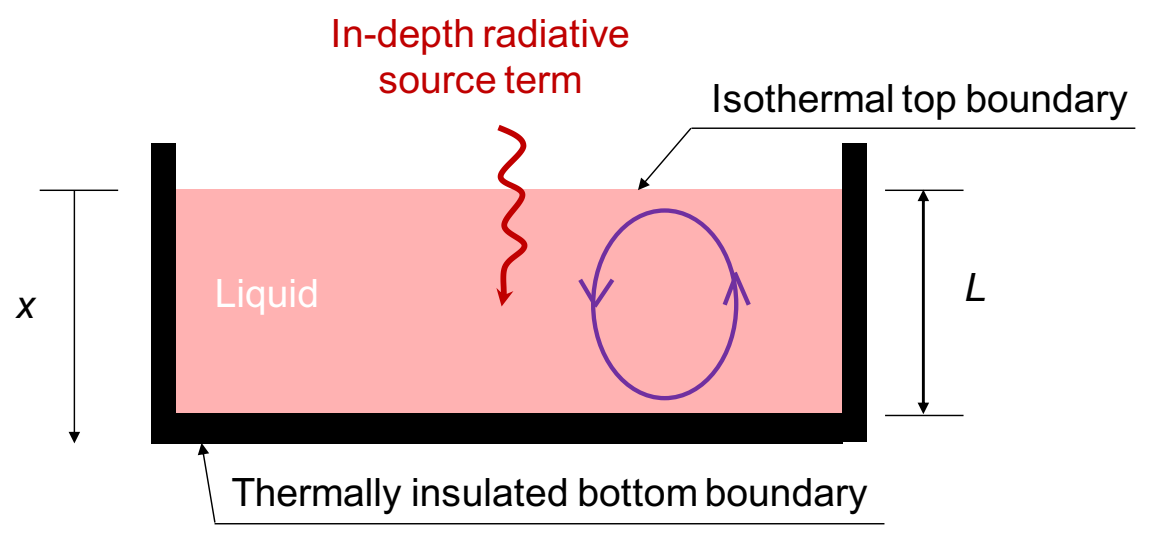

Figure 2 - A schematic of in-depth radiation treatment in pool fires in [3].

In-depth radiation creates a 'volumetric heat source evenly distributed over the liquid layer thickness' (see Fig. 2) and which can be expressed as [3]: 
$\dot{q}_{r}^{\prime \prime \prime}=\frac{\dot{q}_{r, s}^{\prime \prime}}{L}$

where $L$ is the liquid layer thickness.

Such volumetric heat source will generate convective currents which will increase heat transfer within the liquid. This increase in heat transfer is 'indirectly' modeled by increasing the actual thermal conductivity of the liquid, $k$, to the so-called 'effective thermal conductivity', $k_{e f f}$, according to the following equation:

$k_{\text {eff }}=\mathrm{Nu}_{i} \times k$

where $\mathrm{Nu}_{i}$ is the Nusselt number. It is important to mention at this level that we refer here to an 'internal' Nusselt number (within the liquid) and not the Nusselt number used for convective heat transfer at the interface between the liquid and the surrounding gas. In [3], $\mathrm{Nu}_{i}$ is calculated from a correlation for internally heated horizontal plane layer (recall here the volumetric heat source in Eq. (1)) with isothermal top boundary and thermally insulated bottom boundary:

$\mathrm{Nu}_{i}=0.338 \mathrm{Ra}_{i}^{0.227}$

The variable $\mathrm{Ra}_{i}$ denotes the internal Rayleigh number calculated in [3] as:

$\mathrm{Ra}_{i}=\frac{g \beta \dot{q}_{r}^{\prime \prime \prime} L^{5}}{k v \alpha} \frac{\eta^{2}}{Q(\eta)}\left[1-\left(1+\frac{1}{\eta}\right) \exp \left(-\frac{1}{\eta}\right)\right]$

where $g$ is the gravitational acceleration, $\beta, v$ and $\alpha$ are respectively the coefficient of thermal expansion, the kinematic viscosity and the thermal diffusivity of the liquid. The variable $Q(\eta)$ is a normalization constant calculated as:

$Q(\eta)=\eta\left[1-\exp \left(-\frac{1}{\eta}\right)\right]$

and the variable $\eta$ is a normalized length scale associated with the source distribution and calculated as:

$\eta=\frac{1}{\kappa L}$ 
where $\kappa$ is the effective absorption coefficient of the liquid. The latter parameter is calculated using an elaborate algorithm described in detail in [3].

A significant source of uncertainty in the above methodology (and pointed out in [3]) is that the effective thermal conductivity is calculated using the full depth, $L$, of the liquid as a characteristic length scale, see Eq. (6). Whilst this approach may be suitable for a 'relatively thin layer of fuel', it might not be appropriate for deep pools where the convective currents would be localized in the thin top layer of the liquid.

In the next section we propose a novel treatment of in-depth radiation in liquid pool fires.

\subsection{Novel approach}

\subsubsection{Definition of the Rayleigh and Nusselt numbers}

The main idea developed in this work (and originally proposed in [8]) is to use, instead of Eq. (6), the 'classical' definition of the Rayleigh number:

$\mathrm{Ra}_{i}=\frac{g \beta\left(T_{1}-T_{2}\right) \ell_{\text {in-depth }}^{3}}{v \alpha}$

where $T_{1}$ and $T_{2}$ are respectively the bottom and surface temperatures of the fluid layer and $\ell_{\text {in-depth }}$ is a length scale for in-depth radiation.

The effective thermal conductivity in the liquid pool can be calculated using Eq. (4) considering the case of a 'horizontal cavity heated from below' for which the Nusselt number is calculated, instead of Eq. (5), as [9]:

$\mathrm{Nu}_{i}=0.069 \mathrm{Ra}_{i}^{1 / 3} \operatorname{Pr}^{0.074}$

where Pr is the liquid Prandtl number.

As described in [8], the use of Eq. (10) is justified by the fact that in-depth radiation heats up the liquid beneath the surface, leading to the top portion of the liquid (subjected to in-depth radiation) to be hotter at its bottom (i.e., heating from below). This is somewhat counterintuitive, given that the flame is heating the liquid from the top. More details will be provided hereafter. 
In the remainder of the paper, the objective is to develop and explain two methods for the calculation for (1) the length scale and (2) the temperature difference that are used to estimate $\mathrm{Ra}_{i}$ and, consequently, $\mathrm{Nu}_{i}$ and $k_{\text {eff. }}$.

\subsubsection{Analytical solution of the temperature profile}

In the proposed approach we seek to find an analytical solution for heat conduction within the liquid, including in-depth radiation in order to derive an explicit expression for the characteristic length scale that is needed for the calculation of $\mathrm{Ra}_{i}$ and thus, $\mathrm{Nu}_{i}$ and $k_{\text {eff. }}$.

The one-dimensional equation for heat conduction at steady state and including in-depth radiation reads [7]:

$k \frac{\partial^{2} T(x)}{\partial x^{2}}=\frac{\partial \dot{q}_{r}^{\prime \prime}}{\partial x}$

where $\dot{q}_{r}^{\prime \prime}$ is the radiative flux at a given depth $x$. The assumption that the heat flow is unidirectional, in the direction normal to the fuel surface, is mainly based on experimental observations showing that heat loss to the walls is moderate, even for pool diameters as small as $7.4 \mathrm{~cm}$ (e.g., $[5,10])$. The $1 \mathrm{D}$ assumption is most likely to be valid for pool diameters larger than $10 \mathrm{~cm}$.

Based on the classical attenuation law, i.e., Beer's law, the radiative flux is expressed as [7]:

$\dot{q}_{r}^{\prime \prime}=\dot{q}_{r, s}^{\prime \prime} e^{-\kappa x}$

where $\dot{q}_{r, s}^{\prime \prime}$ is the radiative flux at the surface and $\kappa$ is the mean absorption coefficient of the liquid.

Inserting Eq. (12) into Eq. (11) gives:

$k \frac{\partial^{2} T(x)}{\partial x^{2}}=-\kappa \dot{q}_{r, s}^{\prime \prime} e^{-\kappa x}$

When using the boundary conditions $T(x=0)=T_{s}$ and $T(x=L)=T_{b}$, where $L$ is the liquid depth and $T_{s}$ and $T_{b}$ are respectively the top and bottom surface temperatures of the liquid (see Fig. 3), the analytical solution of Eq. (13) reads: 


$$
T(x)=T_{s}+\frac{\dot{q}_{r, s}^{\prime \prime}}{k \kappa}\left(1-e^{-\kappa x}\right)-\frac{x}{L}\left[\left(T_{s}-T_{b}\right)+\left(1-e^{-\kappa L}\right) \frac{\dot{q}_{r, s}^{\prime \prime}}{k \kappa}\right]
$$

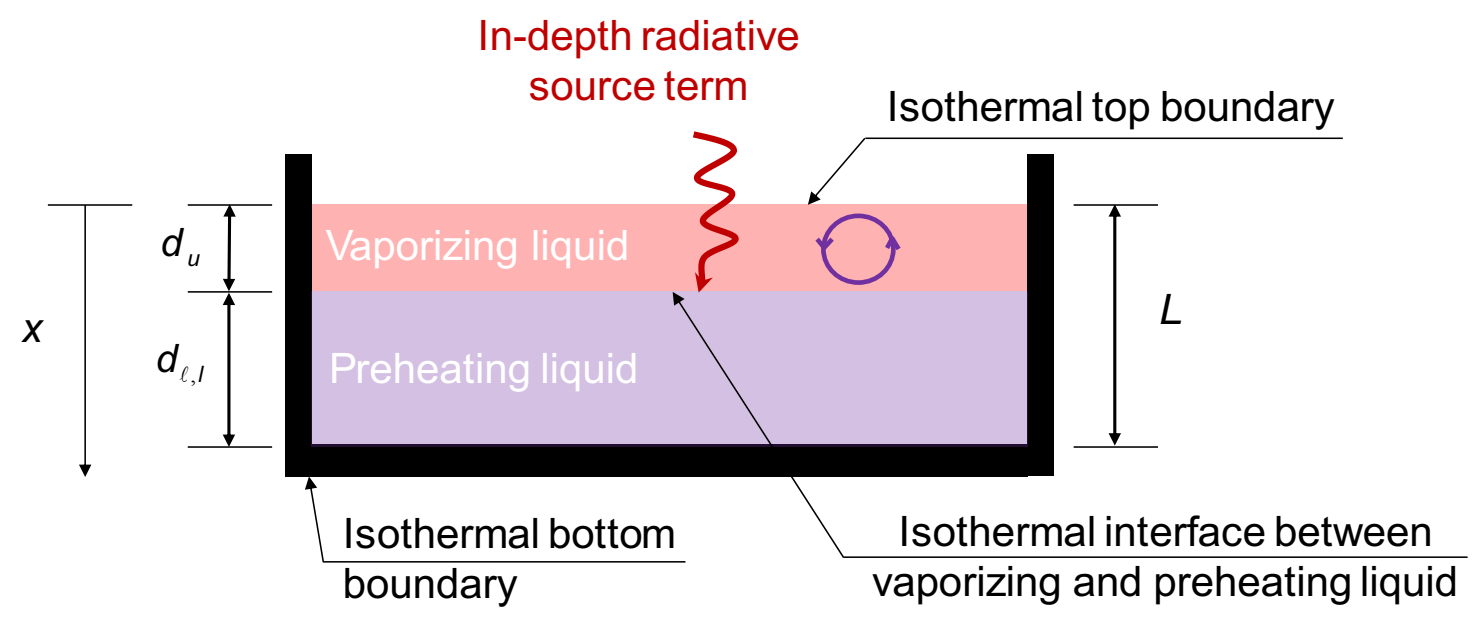

Figure 3 - A schematic of in-depth radiation treatment in pool fires in this work.

An illustration of the obtained liquid temperature profile for one of the cases examined experimentally in [4] is displayed in Fig. 4. As expected, the peak temperature does not occur at the surface but at a specific depth form the surface, yielding a so-called inverse temperature profile' under the effect of in-depth radiation.

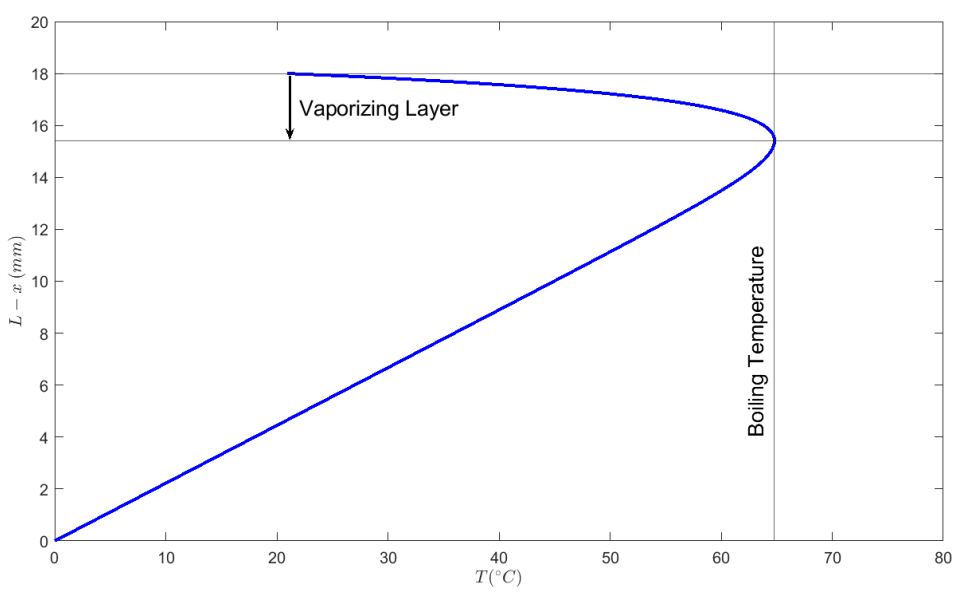

Figure 4 - Liquid temperature profile for a case examined in [4] with the following parameters:

$$
T_{s}=21^{\circ} \mathrm{C}, T_{b}=0^{\circ} \mathrm{C}, \kappa=1000 \mathrm{~m}^{-1}, \dot{q}_{r, s}^{\prime \prime}=12 \mathrm{~kW} / \mathrm{m}^{2}, k=0.2 \mathrm{~W} /(\mathrm{m} . \mathrm{K}) \text { and } L=0.018 \mathrm{~m} .
$$




\subsubsection{Method 1 based on the locus of the peak temperature}

Assuming that the well-mixed layer spans across the liquid layer from the surface up to the location of the peak temperature within the liquid, the analytical expression of $\ell_{\text {in-depth }}$ is calculated as follows:

$\left(\frac{\partial T}{\partial x}\right)_{x=\ell_{\text {in-depth }}}=0$

Applying Eq. (15) for the temperature profile obtained in Eq. (14) gives:

$\ell_{\text {in-depth }}=-\frac{1}{\kappa} \ln \left[\frac{k}{\dot{q}_{r, s}^{\prime \prime} L}\left(T_{s}-T_{b}\right)+\frac{\left(1-e^{-\kappa L}\right)}{\kappa L}\right]$

Note that at depths below $\ell_{\text {in-depth }}$ (i.e., $x>\ell_{\text {in-depth }}$ ) the temperature may remain higher than the surface temperature, as illustrated for example in Fig. 4. Nevertheless, it is monotonically decreasing. Therefore, according to the analytical solution displayed in Eq. (16), at $x>\ell_{\text {in-depth }}$, there are no instabilities that would generate convective currents which occur when a portion of a cold fluid is sitting on top of a hot fluid.

The main aspects that have been developed so far can be visualized in Fig. 3, which gives an overall view of the thermal structure within the liquid and in which $d_{u}=\ell_{i n-d e p t h}$. As displayed in Fig. 3, there is an 'added layer' of complexity in comparison to Fig. 2 in that instead of considering the effect of in-depth radiation using a volumetric source term distributed across the full depth of the liquid, we limit this effect to an upper vaporizing layer whereas the bottom preheating layer will be mainly heated up by thermal conduction from the upper layer and will not be influenced by in-depth radiation. For the sake of completeness and clarity regarding the work described in [3], Fig. 2 refers only to the 'global' treatment of in-depth radiation and does not infer that the liquid is internally thin. In fact, as mentioned earlier, heat transfer is solved in [3] using a 1-D Fourier's equation which is discretized over the full depth of the liquid.

From a mathematical standpoint, it is important to note that Eq. (16) gives a solution to the problem only if the following condition is satisfied: 
$\frac{(k / L) \Delta T}{\dot{q}_{r, s}^{\prime \prime}}<1-\frac{\left(1-e^{-\kappa L}\right)}{\kappa L}$

where $\Delta T=T_{s}-T_{b}$. This is because the sum of the terms between brackets in Eq. (16) (and which is strictly higher than zero) should be strictly lower than 1 so that the natural logarithm gives a negative number and, consequently, $\ell_{\text {in-depth }}>0$, see Eq. (16).

Let us examine now expression (17) from a physical standpoint. If, for given constant values of $\Delta T, L, \dot{q}_{r, s}^{\prime \prime}$ and $\kappa$, the thermal conductivity is 'too high' to satisfy condition (17), this implies that conductive heat transfer 'dominates' radiative heat transfer. Subsequently, there is no onset of instabilities induced by in-depth radiation. The same reasoning could be followed for a 'too high' value of $\Delta T$ or 'too low' values of $L$ (i.e., thin liquid layer) or $\dot{q}_{r, s}^{\prime \prime}$. In fact, it is interesting to rewrite expression (17) as:

$\mathrm{Bi}^{\prime}<\left[1-\frac{\left(1-e^{-\kappa L}\right)}{\kappa L}\right]^{-1}$

where

$\mathrm{Bi}^{\prime}=\frac{\dot{q}_{r, s}^{\prime \prime}}{(k / L) \Delta T}$

is a (so-called here) 'modified' Biot number which, as opposed to the 'classical' Biot number, does not compare the relative importance of convective to radiative heat transfer in terms of resistances, i.e., $\mathrm{Bi}=(L / k) /(1 / h)$, but rather compares the relative importance of thermal radiation with respect to conduction in terms of heat fluxes.

We note also that the condition $d_{\ell, u}<L$ is always satisfied.

Before moving to the assessment of the developed method against experimental data, it is very important to bear in mind two important aspects related to the methodology described above:

1) As opposed to [8], the top surface temperature is not assumed to be the boiling temperature of methanol (i.e., $T_{s}=T_{\text {boiling }}=64.8^{\circ} \mathrm{C}$ [3]), but it is calculated in such a way 
that the peak temperature at $d_{u}=\ell_{\text {in-depth }}$ is equal to $T_{\text {boiling }}$ (see Fig. 4). The purpose is to avoid peak temperatures 'unreasonably' higher than the boiling point if $T_{s}$ is set equal to T boiling.

2) Based on the above, the surface temperature becomes an unknown of the problem. In fact, $T_{s}$ should not be regarded as a 'true' top boundary condition (BC). The latter is imposed by setting a constant radiative heat flux. This is because the main objective in this work is to calculate $\mathrm{Ra}_{i}$ (see Eq. (9)) in order to able to calculate the $\mathrm{Nu}_{i}$ and thus, the effective conductivity. This is done if one is able to estimate the length scale for in-depth radiation and the temperature difference (in the method described above that would be $T_{\text {boiling }}-T_{s}$ ). To sum up, the inputs are $\dot{q}_{r, s}, L, T_{b}$ and the liquid fuel properties and the outputs are $T_{s}$ and $d_{u}=\ell_{\text {in-depth }}$, which are then used to calculate $\mathrm{Ra}_{i}, \mathrm{Nu}_{i}$ and $k_{\text {eff. }}$

Equation (16) (with $d_{u}=\ell_{\text {in-depth }}$ ) is assessed against the experimental data displayed in [4]. The liquid depth, $L$, and the bottom temperature, $T_{b}$, are provided in [4]. The thermal conductivity of methanol is taken as $k=0.2 \mathrm{~W} /(\mathrm{m} . \mathrm{K})$ [3]. The steady-state radiative heat flux incident on the pool surface has not been measured explicitly in [4], but has been estimated in [8] to be $\dot{q}_{r, s}^{\prime \prime}=12 \mathrm{~kW} / \mathrm{m}^{2}$, based on the empirical correlation derived in [11]. The remaining unknown in Eq. (16) is the effective absorption coefficient, $\kappa$. Unfortunately, to the author's best knowledge, there are no direct measurements of $\kappa$ for methanol in the literature. Only measurements for toluene, crude oil, hexane and benzene are reported in [5]. According to [3], although the absorption coefficient spectra was available for methanol, the assumption of blackbody radiation is not applicable. Therefore, the absorption coefficient of ethanol has been used instead. The latter varies between 35 and $1236 \mathrm{~m}^{-1}$, depending on the calculation method (two methods were proposed in [3]) and the path length (which was taken to vary between $1 \mathrm{~mm}$ and $50 \mathrm{~mm}$ ). Given this wide range of values, a sensitivity analysis has been performed on the effective absorption coefficient, $\kappa$. The other properties of methanol required for the calculation of $\mathrm{Nu}_{i}$ are displayed in Table 1. 
Table 1 - Properties of methanol.

\begin{tabular}{|l|l|l|}
\hline Symbol & Name & Value \\
\hline$k$ & Thermal conductivity & $0.20 \mathrm{~W} \cdot \mathrm{m}^{-1} \cdot \mathrm{K}^{-1}$ \\
\hline$\rho$ & Density & $796 \mathrm{~kg} \cdot \mathrm{m}^{-3}$ \\
\hline$c$ & Specific heat & $2.48 \mathrm{~kJ} \cdot \mathrm{kg}^{-1} \cdot \mathrm{K}^{-1}$ \\
\hline$\alpha$ & Thermal diffusivity & $10^{-7} \mathrm{~m}^{2} \cdot \mathrm{s}^{-1}$ \\
\hline$\beta$ & Coefficient of thermal volume expansion & $1.18 \times 10^{-3} \mathrm{~K}^{-1}$ \\
\hline$v$ & Kinematic viscosity & $13.9 \times 10^{-7} \mathrm{~m}^{2} \cdot \mathrm{s}^{-1}$ \\
\hline Pr & Prandtl number & 6.83 \\
\hline$T_{\text {boiling }}$ & Boiling temperature & $64 .{ }^{\circ} \mathrm{C}$ \\
\hline
\end{tabular}

The results displayed in Fig. 5 (left subfigures) for the non-dimensional upper layer depth, i.e., $d_{u}$ $/ L$, show that the 'nearly' constant upper layer depth of about $d_{u}=0.28 \pm 0.03 L$ for $L=18 \mathrm{~mm}$ is best captured with $\kappa=500 \mathrm{~m}^{-1}$. When the bottom temperature $T_{b}$ is between 35 and $50^{\circ} \mathrm{C}$, a value of $\kappa=1000 \mathrm{~m}^{-1}$ gives also a good agreement with the experimental data. In fact, the experimental data for $L=18 \mathrm{~mm}$ is well bounded by the calculations with $\kappa$ between 500 and $1000 \mathrm{~m}^{-1}$ (see Fig. 5a). For $L=12 \mathrm{~mm}$ (see Fig. 5c), a more 'suitable' range for $\kappa$ spans from 300 to $500 \mathrm{~m}^{-1}$. The experimental trend for $L=6 \mathrm{~mm}$ has been more difficult to capture (see Fig. $5 \mathrm{e}$ ) in that a value of $\kappa=500 \mathrm{~m}^{-1}$ appears to be 'suitable' for a bottom temperature $T_{b}$ above $30^{\circ} \mathrm{C}$. For $T_{b}<30^{\circ} \mathrm{C}$, higher values of $\kappa$ (up to $2000 \mathrm{~m}^{-1}$ ) give a better agreement. Nevertheless, the fact that there is no well-mixed upper layer (i.e., $d_{u}=0 \mathrm{~mm}$ ) at $T_{b}=4^{\circ} \mathrm{C}$ could not be captured. One explanation could be that surface tension-driven convection, which becomes important in thin pools, is not considered in the current modelling.

If one performs a linear regression analysis for the experimental and model results for $T_{b}$ between -5 and $50^{\circ} \mathrm{C}$ (as performed in [4] for the experimental lower layer depths) one can see in Table 2 that the 'sensitivity' of $d_{u} / L$ to the bottom temperature increases substantially as the liquid pool gets thinner. This is only 'moderately' captured by the model. It is important to note that Eq. (16) is not a linear but a logarthmic function. Nevertheless, the behaviour of the proposed expression for the range of values examined in this work is 'nearly' linear. 
The results displayed in Fig. 5 (right subfigures) show that $\mathrm{Nu}_{i}$ increases with increasing bottom temperature, $T_{b}$, because of the increased upper layer depth (as shown on the left subfigures), which is directly proportional to $\mathrm{Nu}_{i}$, as can be seen from the combination of Eqs. (9) and (10). Furthermore, the temperature difference (not shown here) used in Eq. (9) does also increase with increasing bottom temperature, $T_{b}$. The right subfigures in Fig. 5 show also that $\mathrm{Nu}_{i}$ increases with decreasing $\kappa$ for similar reasons. Physically speaking, a lower $\kappa$ corresponds to more indepth penetration and thus, more pronounced convective motion. Finally, it is important to mention that the thinner the pool the lower the value of $\mathrm{Nu}_{i}$ because of the reduced buoyancyinduced instability, which is in line with the earlier observation that most likely another mechanism becomes dominant, i.e., surface tension-driven convection.
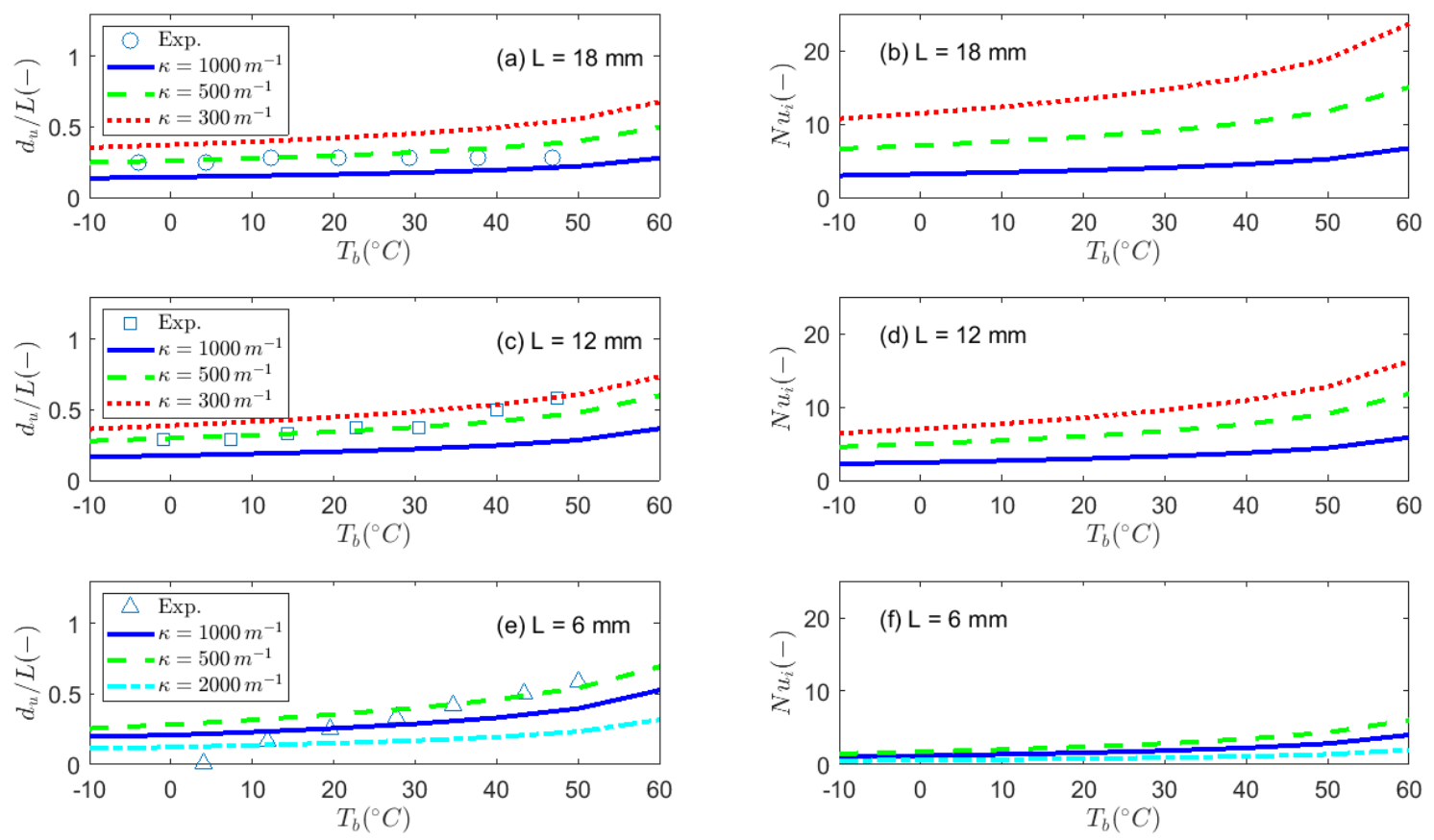

Figure 5 - Results with method 1 for a $90 \mathrm{~mm}$-diameter methanol pool fire as a function of the bottom layer temperature. A comparison between the experimental measurements [4] and numerical predictions of the non-dimensional upper layer depth (left). Predicted $\mathrm{Nu}_{i}$ (right). 
Table 2 - Experimental and model results (with $\kappa=500 \mathrm{~m}^{-1}$ ) of the slope of the linear function

$$
d_{u} / L=f\left(T_{\text {bottom }}\right)
$$

\begin{tabular}{|l|l|l|}
\hline & Exp. data & Model \\
\hline$L=18 \mathrm{~mm}$ & $5.8 \times 10^{-4}\left({ }^{\circ} \mathrm{C}^{-1}\right)$ & $2.4 \times 10^{-3}\left({ }^{\circ} \mathrm{C}^{-1}\right)$ \\
\hline$L=12 \mathrm{~mm}$ & $5.9 \times 10^{-3}\left({ }^{\circ} \mathrm{C}^{-1}\right)$ & $3.2 \times 10^{-3}\left({ }^{\circ} \mathrm{C}^{-1}\right)$ \\
\hline$L=6 \mathrm{~mm}$ & $1.2 \times 10^{-2}\left({ }^{\circ} \mathrm{C}^{-1}\right)$ & $4.6 \times 10^{-3}\left({ }^{\circ} \mathrm{C}^{-1}\right)$ \\
\hline
\end{tabular}

An attempt to 'improve' the current model results is provided in the following sub-section.

\subsubsection{Method 2 based on the radiative heat balance}

The development of the 'radiative heat balance integral method' has started from the observation that using the method illustrated in Fig. 4 for the determination of the upper layer depth, in combination with the constraint on the peak temperature does not exceed the boiling point, inevitably leads to an average upper layer temperature that is significantly below the boiling point. Therefore the idea is to still use the temperature profile provided by Eq. (14), and find the 'optimal' combination of $T_{s}$ and $d_{u}$ that gives and average upper layer temperature that is equal to the boiling point:

$\int_{0}^{d_{u}}\left\{T_{s}+\frac{\dot{q}_{r, s}^{\prime \prime}}{k \kappa}\left(1-e^{-\kappa x}\right)-\frac{x}{L}\left[\left(T_{s}-T_{b}\right)+\left(1-e^{-\kappa L}\right) \frac{\dot{q}_{r, s}^{\prime \prime}}{k \kappa}\right]\right\} d x=T_{b o i l i n g} d_{u}$

An example of the 'transformation' of an 'inverse temperature profile' into the thermal structure described earlier in the paper is given in Fig. 6.

A graphical representation of the optimization procedure is displayed in Fig. 7. Such optimization procedure lies on a root-finding algorithm to find the values of $d_{u}$ and $T_{s}$ which make the curve of the function $f\left(d_{u}\right)=I-T_{\text {boiling }} d_{u}$ tangent with $f\left(d_{u}\right)=0$ at the depth of the vaporizing (boiling) layer. It is very important to recall here that, similarly to method $1, T_{s}$ is an unknown of the problem that does not represent the actual boundary condition at the liquid surface (which is the radiative heat flux). The difference with method 1 is that the peak temperature is not fixed to $T_{\text {boiling }}$ but is an output of the algorithm. As shown in Fig. 6, the peak temperature exceeds the boiling temperature so that the average temperature across the upper 
layer becomes equal to $T_{\text {boiling. }}$ To sum up, the inputs are $\dot{q}_{r, s}, L, T_{b}$ and the liquid fuel properties and the outputs are $T_{s}$, the peak temperature and $d_{u}$, which are then used to calculate $\mathrm{Ra}_{i}, \mathrm{Nu}_{i}$ and $k_{\text {eff. }}$

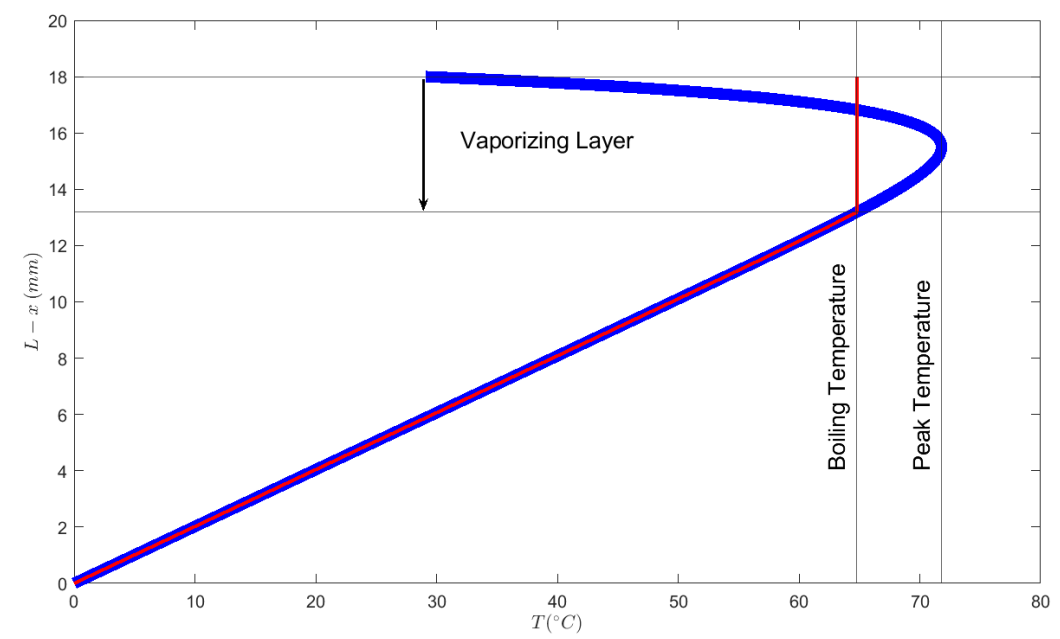

Figure 6 - Liquid temperature profile for a case examined in [4] with the following parameters: $T_{s}=29.1^{\circ} \mathrm{C}, T_{b}=0{ }^{\circ} \mathrm{C}, \kappa=1000 \mathrm{~m}^{-1}, \dot{q}_{r, s}^{\prime \prime}=12 \mathrm{~kW} / \mathrm{m}^{2}, k=0.2 \mathrm{~W} /(\mathrm{m} . \mathrm{K})$ and $L=0.018 \mathrm{~m}$. The thick blue line represents the profile from Eq. (14) and the thin red line represents the temperature profile obtained by applying the suggested 'radiative heat balance integral method'.

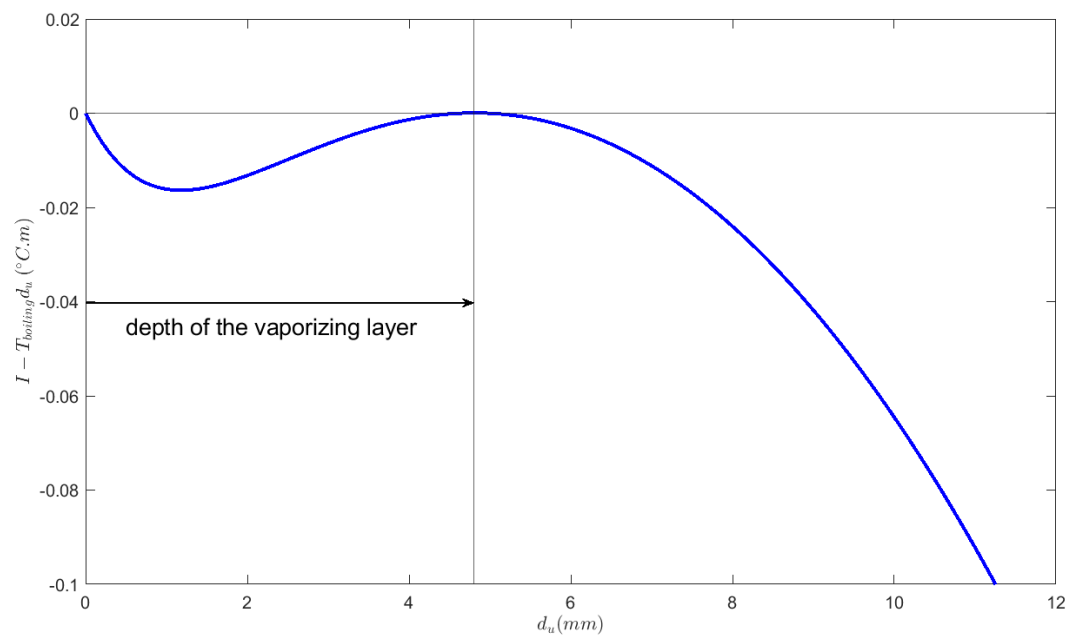

Figure 7 - A graphical representation of the optimization procedure used in method 2 for the same case displayed in Fig. 6. The parameter $I$ in the $y$-axis represents the right-hand side in Eq. (20). 
One can state, based on the argument developed in the first method, that below the locus of the peak temperature in the profile provided by Eq. (14) (see blue thick line in Fig. 6) there are no instabilities that would lead to convective motion and mixing. Nevertheless, it is important to bear in mind that the interface located at the position provided by $\ell_{\text {in-depth }}$, see Eq. (16), is not a rigid interface (boundary) and as such it is reasonable to assume that the convection motion and mixing extends below it. This is the first main difference between method 1 (Eq. (16)) and method 2 (Eq. (20)) for the determination of the upper layer depth. The second difference lies in the determination of the temperature difference in the expression of the Rayleigh number, Eq. (9). In the first method, $T_{s}$ is an unknown and the peak temperature is set equal to the boiling temperature, whereas in the second method both temperatures are unknown. Furthermore, method 2 has the advantage of yielding, by definition, the thermal structure described earlier. However, method 1 has the advantage of giving an explicit expression (along with the constraint that the peak temperature is equal to the boiling point), as opposed to the iterative 'optimization' procedure required in method 2 .

Figures 8a, 8c and 8e show that a value of $\kappa=1000 \mathrm{~m}^{-1}$ gives an overall good agreement with the experimental data for the non-dimensional upper layer depth, $d_{u} / L$, particularly for $L=12 \mathrm{~mm}$. For $L=18 \mathrm{~mm}$, the upper layer depth is overpredicted with $\kappa=1000 \mathrm{~m}^{-1}$ for $T_{b}>30^{\circ} \mathrm{C}$. For $L=$ $6 \mathrm{~mm}$ and $T_{b}<35^{\circ} \mathrm{C}$, the absorption coefficient had to be increased to 2000 and even $5000 \mathrm{~m}^{-1}$ to have a good agreement with the experimental data. Nevertheless, as mentioned for method 1, this can be attributed to the fact that surface tension-driven convection, which becomes important in thin pools, is not considered in the current modelling. The main difference in the upper layer depth results between the two methods is in the value of $\kappa$ which gives the overall best results: $\kappa$ $=500 \mathrm{~m}^{-1}$ for method 1 and $\kappa=1000 \mathrm{~m}^{-1}$ for method 2 .

Regarding the predictions for $\mathrm{Nu}_{i}$, the overall same trends are observed for method 2 as for method 1. A more detailed comparison is provided and discussed in the next section. 

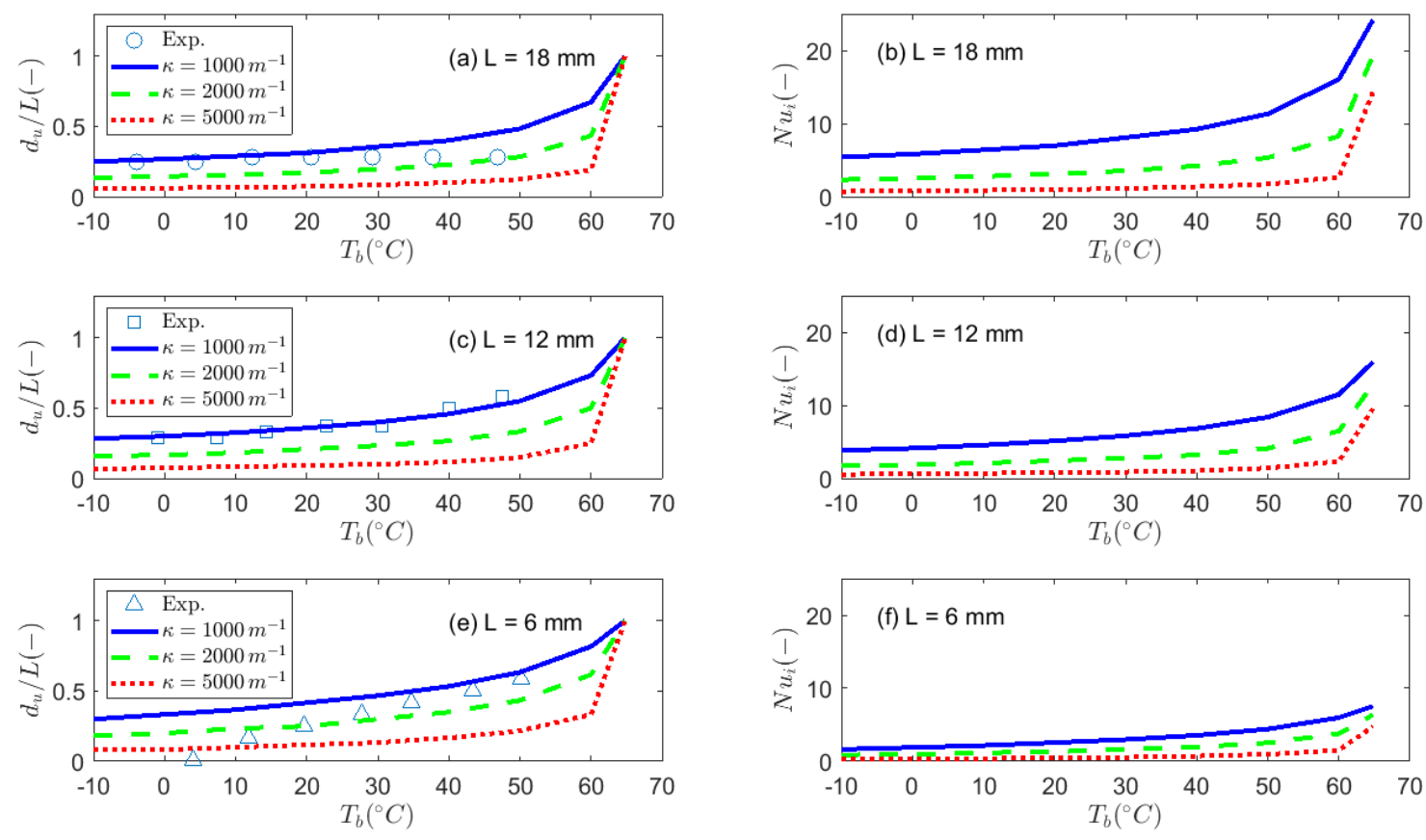

Figure 8 - Results with method 2 for a $90 \mathrm{~mm}$-diameter methanol pool fire as a function of the bottom layer temperature. A comparison between the experimental measurements [4] and numerical predictions of the non-dimensional upper layer depth (left). Predicted $\mathrm{Nu}_{i}$ (right).

Before comparing the method proposed in [4] with the two methods proposed in this work, a sensitivity analysis has been carried out on the radiative heat flux, which was taken in the calculations above as $\dot{q}_{r, s}^{\prime \prime}=12 \mathrm{~kW} / \mathrm{m}^{2}$. In Fig. 9, the results are displayed (for $L=12 \mathrm{~mm}$ and $\kappa$ $=1000 \mathrm{~m}^{-1}$ using method 2) with values that are $25 \%$ higher and lower. It appears that the predictions of the non-dimensional upper layer depth and $\mathrm{Nu}_{i}$ are not very sensitive to the considered variations in the radiative heat flux. 

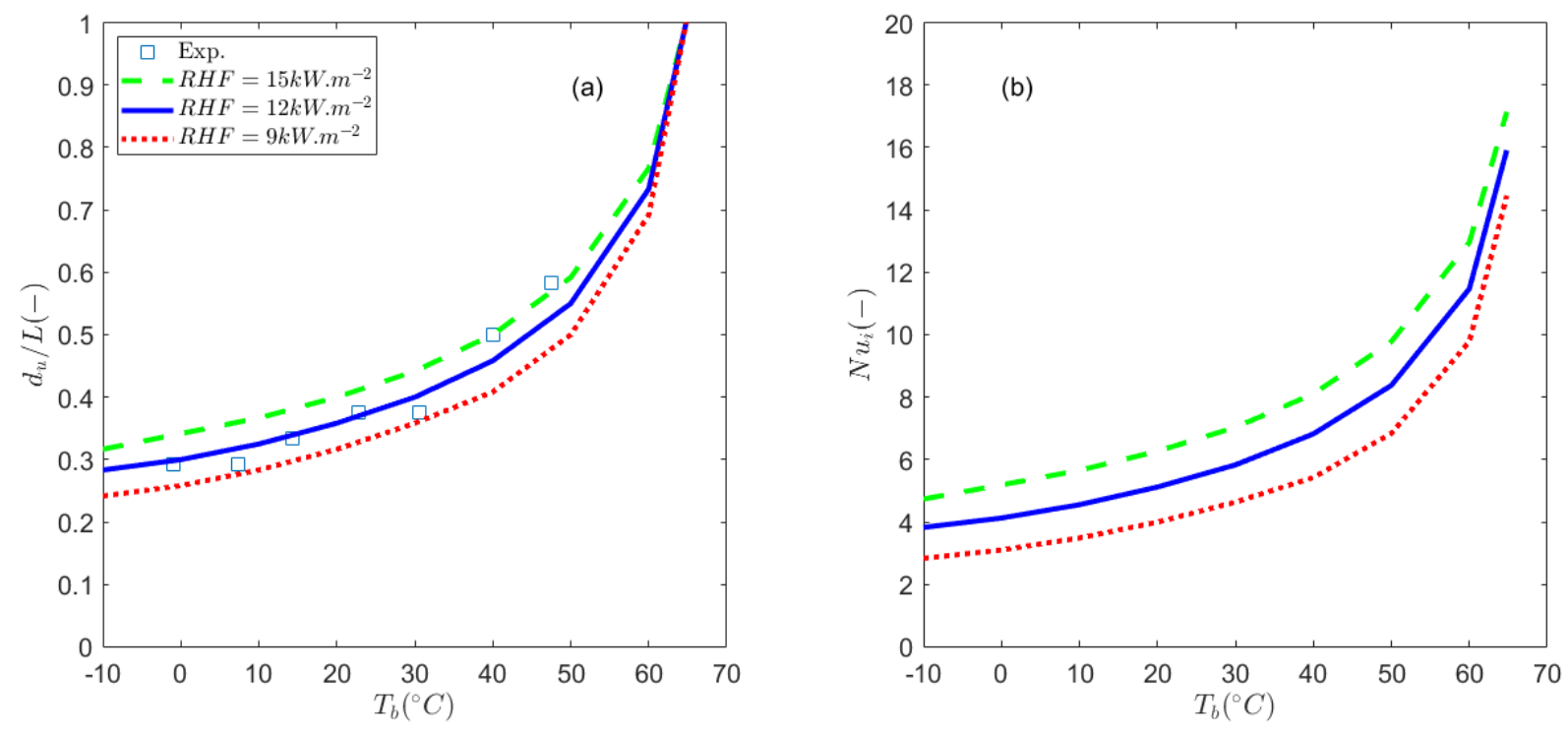

Figure 9 - Influence of the radiative heat flux on the predictions (using method 2) of (a) the nondimensional upper layer depth and (b) the Nusselt number, $\mathrm{Nu}_{i}$, for the $90 \mathrm{~mm}$ - diameter methanol pool fire with $L=12 \mathrm{~mm}$ and $\kappa=1000 \mathrm{~m}^{-1}$.

\subsubsection{A comparative analysis}

In order to compare the two methods proposed here with the approach proposed in [3], let us consider the case of a methanol liquid fuel subjected to a radiative heat flux of $20 \mathrm{~kW} / \mathrm{m}^{2}$ (at its upper surface) with depths ranging from $0.005 \mathrm{~m}$ to $0.200 \mathrm{~m}$. The temperature at the bottom boundary is assumed to take the value of $T_{b}=20^{\circ} \mathrm{C}$.

The Nusselt numbers displayed in Fig. 10a show significant deviations between the method proposed in this work and the method developed in [3]. The deviations increase with increased liquid depth. This is caused by the difference in depth over which the in-depth radiative source term is distributed, as visualized by comparing Figs. 2 and 3. This is also shown in Fig. 10b where the upper layer depth, $d_{u}$, predicted by the two methods is significantly lower than the overall liquid depth, $L$, used as a length scale in Eq. (6). When comparing the results between method 1 and method 2, it is important to bear in mind that the same absorption coefficient of $\kappa$ $=1000 \mathrm{~m}^{-1}$ has been used here. Based on the sensitivity analysis carried out earlier in this work on $\kappa$ for the two methods, we recall here that for the same value of $\kappa$, method 1 predicts lower 
values of $d_{u}$, as confirmed here in Fig. 10b. This is the main reason for the discrepancies between method 1 and method 2 in the values of $\mathrm{Nu}_{i}$, the calculated temperature differences used in Eq. (9) (and not shown here) are rather similar.
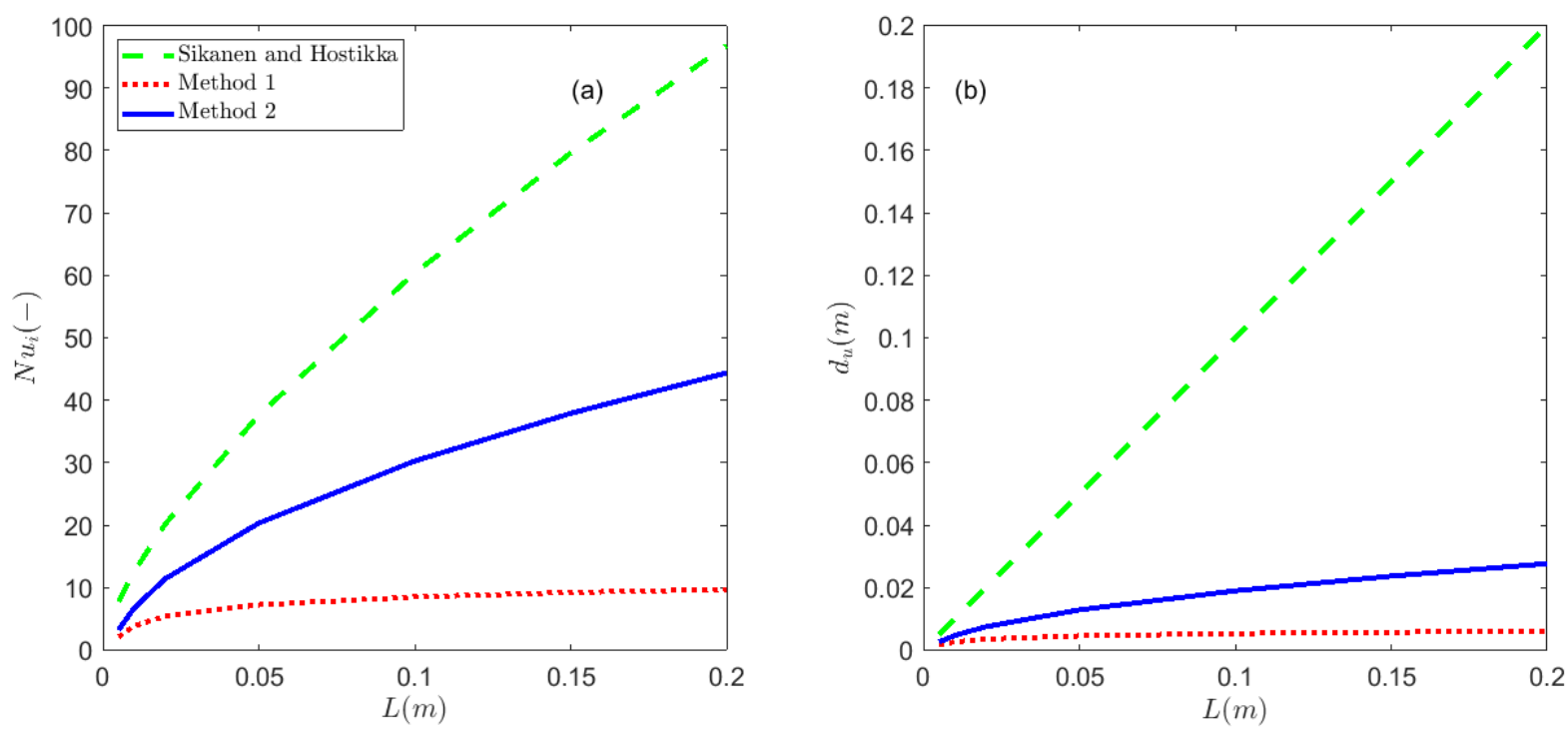

Figure 10 - A comparison between the approach proposed in [3] and the two methods developed in this work. The liquid fuel is methanol, $T_{b}=20^{\circ} \mathrm{C}, \kappa=1000 \mathrm{~m}^{-1}$ and $\dot{q}_{r, s}^{\prime \prime}=20 \mathrm{~kW} / \mathrm{m}^{2}$.

At this stage, it is difficult to be assertive with respect to the 'validity' of the calculated Nusselt numbers or the 'performance' of one calculation procedure over the other. More detailed experimental measurements are needed for this matter. The same comment holds for an important fuel property used herein, namely the effective absorption coefficient, $\kappa$. Nevertheless, in the meantime, one could implement the theoretical approach described herein in the simulation of pool fires and assess its influence on global quantities such as the peak (and steady) heat release rate and the time to reach the peak (or the steady-state).

\section{Foreseen applications in numerical modelling}

It is envisioned that the theoretical and analytical work developed here can serve as a sub-model in the modelling of the liquid heat-up in the case of a pool fire. More specifically, the 1-D Fourier's equation would read: 
$\rho c \frac{\partial T}{\partial t}=\frac{\partial}{\partial x}\left(k \frac{\partial T}{\partial x}\right)$ with $\left\{\begin{array}{l}k=k_{\text {eff }} \text { if } x \leq d_{u} \\ k=k \text { if } x>d_{u}\end{array}\right.$

Besides the calculation procedure of $k_{\text {eff, }}$ there are two differences with the work described in [3]. The first one is that the radiative heat flux would exclusively be used as a boundary condition for the upper (thermally thin) layer and not as a source term in Eq. (1). The second difference between Eqs. (1) and (21) is related to the treatment of in-depth radiation as one can visualize by comparing Figs. (2) and (3). The increased conductivity in Eq. (21) is only applied to the upper layer. Equation (20) plays in this regard a key role in the calculation procedure.

An alternative to solving Eq. (21) would be to use the concept of two-zone modelling for heat transfer in the liquid phase. In such two-zone modelling framework, the volume of the two 'control volumes' would be known thanks to Eq. (16) or (20). A remaining aspect to be modeled (regarding heat transfer) is the heat exchange between the two zones (i.e., the upper and the lower layer), which is out of scope of the current paper.

Both ways of liquid heat-up modelling described above (i.e., 1-D Fourier's equation or two-zone modelling) can be coupled to gas phase modelling in CFD simulations with the intent of saving computational resources without compromising the accuracy of the simulations, which is, in any case, not 'guaranteed' even if the full Navier-Stokes equations in the liquid phase are solved.

\section{Conclusion}

Fully predictive numerical simulations of liquid pool fires require a full and accurate coupling between the gas phase and the liquid phase. Whilst extensive research work has been (and is still being) carried out on the gas phase, focusing on aspects such as combustion, soot and thermal radiation, an accurate treatment of the liquid heat-up is still lacking.

The purpose of the current work has been to explore a methodology for the modelling of the liquid phase that would not require extensive and prohibitive computational resources to perform 'practical' fire dynamics simulations.

The theoretical and analytical work carried out herein is based on the assumption that in-depth radiation generates hydrodynamic (Rayleigh-Bénard) instabilities leading to enhanced 
convective heat transfer over a limited upper layer depth of the liquid. The analytical expression that has been derived for the upper layer depth (under the aforementioned assumption) has been compared against available experimental data for a $9 \mathrm{~cm}$-diameter methanol steady-state pool fire and three different liquid depths $(18,12$ and $6 \mathrm{~mm})$, maintaining the bottom boundary temperature at several values between -10 and $50^{\circ} \mathrm{C}$. Encouraging results have been obtained in terms of qualitative variation of $d_{u}$ with the bottom layer temperature (or temperature difference between top and bottom) the overall liquid depth. However, this method does not ensure a wellmixed upper layer at a temperature near the boiling point, as suggested by the experimental data. Subsequently, an improvement is proposed based on a radiative heat balance integral method. In addition to the above, a novel methodology is developed for the calculation of the 'effective' thermal conductivity as a means to circumvent detailed calculations of heat transfer within the liquid.

The theoretical and analytical work presented in this paper requires further validation by considering a wider range of conditions (e.g., fuel type and pool diameter). It has the potential to strongly support CFD simulations that rely on (1) a 1-D heat transfer treatment of the liquid using a discretized Fourier's equation (as in [3]) or (2) a novel treatment of the liquid heat-up using a two-zone modelling in an analogous way to what is commonly done about smoke stratification in the early stages of an enclosure fire.

\section{References}

[1] Maragkos G, Beji T, Merci B. Advances in modelling CFD simulations of turbulent gaseous pool fires. Combust Flame 2017; 181: 22-38.

[2] Fukumoto K, Wen JX, Li M, Ding Y, Wang C. Numerical simulation of small pool fires incorporatig liquid fuel motion. Combust Flame 2020; 213: 441-454.

[3] Sikanen T, Hostikka S. Modelling and simulation of liquid pool fires with in-depth radiation and heat transfer. Fire Saf J 2016; 80: 95-109.

[4] Vali A, Nobes DS, Kostiuk LW. Fluid motion and energy transfer within burning liquid fuel pools of various thicknesses. Combust. Flame 2015; 162: 1477-1488. 
[5] Inamura T, Saito K, Tagavi KA. A Study of Boilover in Liquid Pool Fires Supported on Water. Part II: Effects of In-depth Radiation Absorption. Combust Sci Technol 1992; 86: 105-119.

[6] Hayasaka H. Unsteady Burning Rates of Small Pool Fires. In: Hasemi, Y. (Ed.), Fire Safety Science - Proceedings of the Fifth International Symposium, pp, 499-510, 1997.

[7] Garo JP, Gillard P, Vantelon JP. On The Thin Layer Boilover. In: Curtat, M. (Ed.), Fire Safety Science - Proceedings of the Sixth International Symposium, pp, 579-590, 2000.

[8] Beji T, Merci B. Analytical Modelling of the Effect of In-Depth Radiation Within a Liquid Layer in the Case of a Pool Fire. The $9^{\text {th }}$ International Seminar on Fire and Explosion hazards, $21-26$ April 2019, Saint-Pietersburg, Russia.

[9] Incropera FP, DeWitt DP. Fundamentals of Heat and Mass Transfer, 4th ed., New York, , John Wiley \& Sons, 1996.

[10] Zaho J, Huang H, Jomaas G, Zhong M, Yang R. Experimental study of the burning behaviors of thin-layer pool fires. Combust. Flame 2018; 193: 327-334.

[11] Ditch BD, de Ris JL, Blanchat TK, Chaos M, Bill Jr. RG, Dorofeev SB. Pool fires - an empirical correlation. Combust. Flame 2013; 160 (12): 2964 - 2974. 